\title{
Progress in dynamic EBSD pattern simulation
}

\author{
G. Nolze*, A. Winkelmann** \\ * Bruker Nano, Schwarzschildstr. 12, D-12489 Berlin, Germany \\ ** Max-Planck-Institute of Microstructure Physics, Weinberg 2, D-06120 Halle (Saale), Germany
}

EBSD is nowadays a common technique for the characterization of crystalline microstructures in scanning electron microscopy. The diffraction patterns are often interpreted by superimposing individual Kikuchi bands which are geometrically described by band edges derived from Braggs law. For the typically very simple crystal structures of technically applied materials, such a simplification of the Kikuchi pattern interpretation works sufficiently well, especially for orientation determinations as a main application of EBSD. The more complex crystal structures, however, are a challenge for EBSD indexing routines which in such cases often fail unpredictably. The use of only the intensities of single reflectors for a description of the Kikuchi band intensity and as a cut-off criterion for a pre-selection of the strongest bands are not satisfactory. Often the result will match too many phases, or there are certain deviations in the intensity prediction which must be adapted manually. This is already problematic if one is absolutely sure that the patterns are originating from the expected phase and it becomes a very questionable procedure for an unknown phase.

Alternative approaches use information about the band widths and make possible a rough estimation of the reduced unit cell dimensions. This helps if the lattice parameters are very different. Moreover, a combination with EDS is helpful if the distance between adjacent spots is in the scale of the EDS resolution, i.e. microns, because of the much higher interaction volume of this technique. In contrast, for high resolution maps, the phase separation by EDS is quite complicated because of the matrix effect. This effect leads to a detection of EDS signal coming mostly from much larger depths than the EBSD diffraction signal. Summarizing, there is a clear need for an implementation of an improved intensity description of EBSD signals. Many practical applications will benefit from a correct pattern simulation. This includes phase identification and strain determination with the need for reference patterns, multiphase mapping, and pseudo-symmetry investigations. But also orientation measurements may profit from improved simulations via a more reliable intensity prediction compared to the currently used reflector ranking based on kinematic theory and X-ray databases.

However, the local intensity distribution within an EBSD pattern is difficult and time-consuming [1] to calculate since the main assumptions of the kinematic theory are not fulfilled and dynamic electron diffraction theory has to be used. This is why it is not surprising that the commonly used reverse-drawn edges of the most intense reflectors, as shown in Fig. 1b), cannot really convince. Especially when only the geometrical simulation is used, it is often not simple to judge whether the solution is correct because of the large number of bands that is, in principle, allowed to be observed. The EBSD pattern is much better described considering dynamic diffraction theory as is shown in Fig. 1d). The agreement with the experimental pattern is convincing, although the contrasts in the simulation are stronger due to several experimental influences which tend to weaken the observed contrast and which are not accounted for in the present theory.

Using the newly developed software as part of the analytical software package ESPRIT, the simulation time for a crystalline phase using dynamic EBSD theory could be extremely reduced, down to minutes on standard PCs. In combination with standard databases, the program allows dynamic EBSD simulations for any crystallographic phase, including import of general CIF files. The software allows real-time rotation of spherical EBSD patterns [2] and of their gnomonic and stereographic projections. In this way, after the simulation of the complete intensity distribution, the pat- 
tern for arbitrary orientations can be drawn interactively in real-time. The extracted line profiles can be used for a more realistic intensity rating of Kikuchi bands.

\section{References}

[1] A. Winkelmann et al., Ultramicroscopy, 107 (2007) 414.

[2] A. Day, J.Microscopy, 230 (2008) 472.

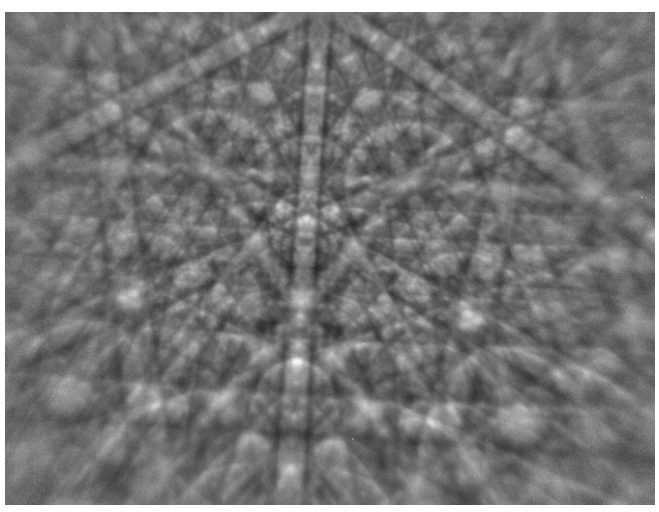

a) $\mathrm{W}_{2} \mathrm{C}$ experiment

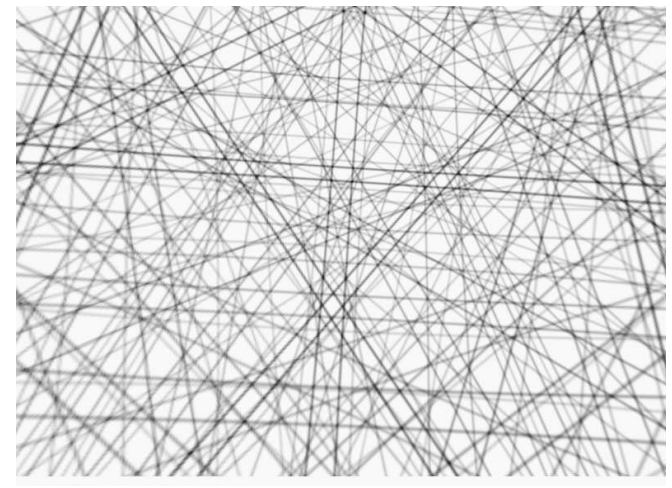

c) kinematic simulation

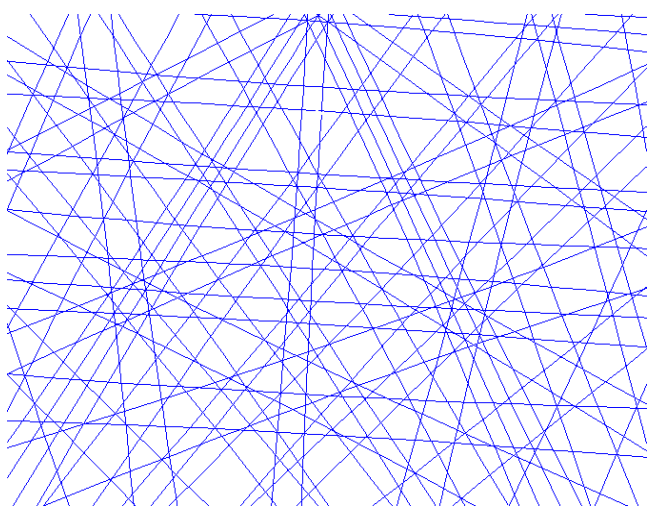

b) geometric interpretation

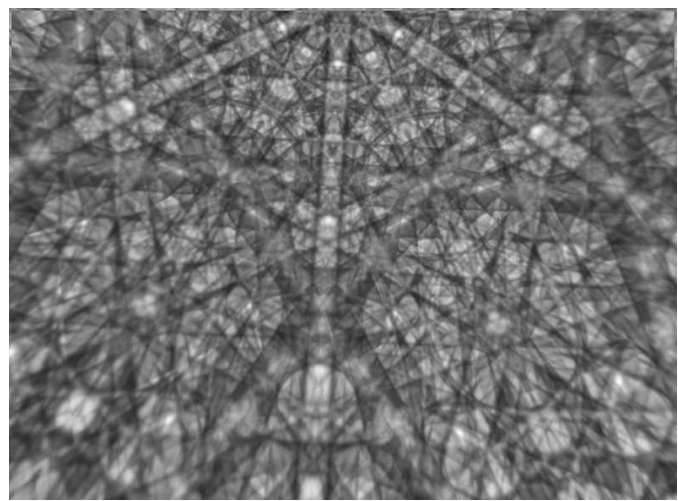

c) dynamic simulation

\section{Fig. 1}

a) the experimental $\mathrm{W}_{2} \mathrm{C}$ EBSD pattern, b) geometrical prediction of band edges using Braggs Law c) kinematical simulation with intensity scaling of band edges based on structure factor, d) full dynamic simulation for $\mathrm{W}_{2} \mathrm{C}$. 